\title{
Pharmaceutical Quality/CMC Batch Utilization Terminology
}

National Cancer Institute

\section{Source}

National Cancer Institute. Pharmaceutical Quality/CMC Batch Utilization Terminology.

NCI Thesaurus. Code C133846.

A category of terminology used to qualify the information pertaining to batch utilization in the framework of the Pharmaceutical Quality/Chemistry, Manufacturing and Controls documents. 